\title{
TRANSFORMATION RULES FOR BONDI INTEGRATION FUNCTIONS
}

\author{
(Presented by G. Kuzmin)
}

\section{Introduction}

For asymptotically flat space-times in general relativity there are several possibilities to define energy-momentum and angular momentum. These definitions are mostly equivalent; however, a common difficulty arises if we consider their transformation properties under the Bondi-MetznerSachs (BMS) group, the generally accepted symmetry group for asymptotically flat space-times. No problems arise in connection with energymomentum, but as angular momentum depends on supertranslations further analysis is needed. The usual solution to the problem is obtained by restricting the BMS group to the Poincaré group. It can be easily done in nonradiative cases, but even then the result is doubtful [ ${ }^{1}$ ]. In radiative spaces the necessity to eliminate supertranslations has led to the construction of complex «heaven spaces» $\left[{ }^{2}\right]$.

Our aim is to examine the possibility of understanding the physical meaning of transformation properties of angular momentum within the framework of traditional general relativity, retaining the full BMS group. This problem will not be given a final solution in the present paper. When dealing with the problem; we first discover that complete transformation rules can be found nowhere, probably because of their rather lengthy and non-elegant appearance. So the task of the present paper will be to present the transformation rules as compactly as possible. This is done in Sec. 3. In Sec. 4 we shall prove that our transformation forms a representation of the BMS group, using formalism developed in
Sec. 2 .

We start by presenting the BMS group in a form convenient for the further use.

\section{Asymptotic solution and the method of approaching the BMS group}

All information concerning total energy, momentum and angular momentum is contained in the Bondi integration functions $M, N$ and $c$ which are introduced when the Einstein equations are integrated in the Bondi coordinates; they define the metric tensor in the power series of $r^{-1}\left[{ }^{3}\right]$ :

$$
\begin{aligned}
& g_{00}=1-2 M / r+O\left(r^{-2}\right), \\
& g_{01}=1-c \bar{c} / 2 r^{2}+O\left(r^{-3}\right) \\
& g_{0 \Omega}=\partial c+2\left\{N-\frac{1}{4} \tilde{\partial}(c \bar{c})\right\} / r+O\left(r^{-2}\right), \\
& g_{22}+g_{33} / \sin ^{2} \vartheta=-2 r^{2}-4 c \bar{c}+O\left(r^{-1}\right), \\
& g_{\Omega \Omega}=-4 c r+O\left(r^{-1}\right) .
\end{aligned}
$$


Here bar above $c$ denotes the complex conjugate. The definitions and some properties of edth operators $\partial$ and $\tilde{\partial}$ are given in Appendix A. The index $\Omega$ is used to combine two angle components of a tensor into a complex one, defined as $A_{\Omega}=A_{2}-\mathrm{i} A_{3} / \sin \vartheta$, i.e.

$$
\begin{aligned}
& g_{0 \Omega}=g_{02}-\mathrm{i} g_{03} / \sin \vartheta, \\
& g_{\Omega \Omega}=g_{22}-2 \mathrm{i} g_{23} / \sin \vartheta-g_{33} / \sin ^{2} \vartheta .
\end{aligned}
$$

We shall use standard notation for generalized Bondi coordinates, $u, r, \vartheta, \varphi$ standing for retarded time, radial coordinate and angle variables, respectively. The Bondi coordinates are fixed by the following coordinate conditions

$$
\begin{aligned}
& g_{11}=g_{12}=g_{13}=0, \\
& g_{22} g_{33}-g_{23} g_{23}=r^{4} \sin ^{2} \vartheta, \\
& g_{01}=1+O\left(r^{-1}\right) .
\end{aligned}
$$

The infinitesimal coordinate transformations $\delta x^{i}$, leaving conditions (1.2) satisfied form the Lie algebra of the BMS group. Introducing a complex notation

$$
\delta \Omega=\delta \vartheta-i \sin \vartheta \delta \varphi,
$$

the coordinate transformations can be expressed by a real function $\tau$ and a complex function $\omega$,

$$
\begin{aligned}
& \delta u=\tau(u, \vartheta, \varphi), \\
& \delta \Omega=\omega(\vartheta, \varphi)+O\left(r^{-1}\right),
\end{aligned}
$$

where $\tau$ and $\omega$ are restricted by

$$
\begin{aligned}
& \frac{\partial \omega}{\partial u}=\tilde{\partial}_{-1 \omega}=0, \\
& \partial_{-1} \omega+\tilde{\partial}_{+1} \bar{\omega}=-4 \frac{\partial \tau}{\partial u} .
\end{aligned}
$$

As $u$-independent part of $\tau, \tau_{0}(\vartheta, \varphi)$ remains arbitrary; the BMS group includes an infinite parameter subgroup called supertranslations. The dimensionality of this subgroup may be reduced to four by further restriction on $\tau, \partial \partial \tau=0$. The latter, together with restrictions (1.5), defines exactly the Poincaré group.

Let us recur to (1.5) as it stands. The customary next step would be to find all linearly independent solutions of (1.5), each corresponding to a one-parameter subgroup of the BMS group and defining a generator. Contrary to this; we want to retain $\tau$ and $\omega$ as basic variables defining the group via (1.5), for the reason that through them the desired transformation rules for $N, M, c$ may be presented in the most compact form. Such a formulation is somewhat similar to that of B. S. DeWitt $\left.{ }^{4}\right]$ with an important difference that our $\tau$ and $\omega$ are not independent real functions as they should be to enable us to define the structure constants of the group. To deal with the case, in the next section we shall introduce the basic commutation relation in a new form that enables us to prove that transformation is a representation without structure constants being used.

Ordinary formalism may be established at any stage of our treatment if proper values of $\tau, \omega$ are substituted; the required formulae are given in Appendix B. 


\section{The basic commutation relation for a representation of the group}

Let us consider an $m$-dimensional manifold $M$ that possesses some symmetry. It may happen that the symmetry group $G$ can be locally defined in terms of allowable transformations of somehow chosen («pre-
ferred») coordinates:

$$
\begin{aligned}
& x^{\prime \mu}=x^{\mu}+\varepsilon^{\mu}(x), \\
& L^{k} \varepsilon^{\mu}=0, \\
& k=
\end{aligned}
$$

where $\varepsilon^{\mu}$ are infinitesimal and $L_{\mu}$ are some operators.

In addition; let there be a collection of $n$ functions $f_{A}(x)$ on which (2.1) generates a transformation

$$
\delta f_{A}(x)=S_{A B}(\varepsilon) f_{B}(x),
$$
where $S_{A B}(\varepsilon)$ are linear operators. In matrix notation it may be
written as

$$
\delta f=S(\varepsilon) f .
$$
The infinitesimal operator $S$ forms a representation of the group if and
only if

$$
\left[S\left(\varepsilon_{2}\right), S\left(\varepsilon_{1}\right)\right]=S\left(q\left(\varepsilon_{1}, \varepsilon_{2}\right)\right),
$$

where $q\left(\varepsilon_{1}, \varepsilon_{2}\right)$ denotes the commutator of $\varepsilon_{1}$, $\varepsilon_{2}$, i. e. the result of successive transformations

$$
\begin{array}{cc}
x^{\prime \mu}=x^{\mu}+\varepsilon_{1}^{\mu}(x), & x^{\prime \prime \mu}=x^{\prime \mu}+\varepsilon_{2}^{\mu}\left(x^{\prime}\right), \\
x^{\prime \prime \prime} \mu=x^{\prime \prime \mu}-\varepsilon_{1}^{\mu}\left(x^{\prime \prime}\right) ; & x^{\prime \prime \prime \prime} \mu=x^{\prime \prime \prime} \mu-\varepsilon_{2}{ }^{\mu}\left(x^{\prime \prime \prime}\right) \\
q^{\mu}\left(\varepsilon_{1}, \varepsilon_{2}\right)=x^{\prime \prime \prime} \mu-x^{\mu} . &
\end{array}
$$

The commutator $q$ may be easily calculated

where

$$
\begin{aligned}
q^{\mu}\left(\varepsilon_{1}, \varepsilon_{2}\right) & =\varepsilon_{1}{ }^{\sigma} \frac{\partial}{\partial x^{\sigma}} \varepsilon_{2}^{\mu}-\varepsilon_{2}{ }^{\sigma} \frac{\partial}{\partial x^{\sigma}} \varepsilon_{1}^{\mu} \\
& =S_{0}\left(\varepsilon_{2}\right) \varepsilon_{1}^{\mu}-S_{0}\left(\varepsilon_{1}\right) \varepsilon_{2}{ }^{\mu},
\end{aligned}
$$

$$
S_{0}(\varepsilon)=-\varepsilon^{\sigma} \frac{\partial}{\partial x^{\sigma}}
$$

is the generator for a scalar.

With (2.6) the basic relation (2.5) becomes

$$
\left[S\left(\varepsilon_{1}\right), S\left(\varepsilon_{2}\right)\right]=S\left(S_{0}\left(\varepsilon_{1}\right) \varepsilon_{2}-S_{0}\left(\varepsilon_{2}\right) \varepsilon_{1}\right) .
$$

As $S(\varepsilon)$ is linear in its argument, $(2.8)$ is equivalent to

$$
S\left(\varepsilon_{1}\right) S\left(\varepsilon_{2}\right)-1 \leftrightarrow 2=S\left(S_{0}\left(\varepsilon_{1}\right) \varepsilon_{2}\right)-1 \leftrightarrow 2
$$

For practical use the last formulation is more suitable as it allows to make calculations modulo symmetric terms in $\varepsilon_{1}, \varepsilon_{2}$.

Condition (2.8) or (2.9), for each $\varepsilon_{1}, \varepsilon_{2}$ satisfying (2.2), is equivalent to customary condition for each pair of generators $S_{m}, S_{n}$

$$
\left[S_{m}, S_{n}\right]=c_{m n}{ }^{p} S_{p}
$$

where $c_{m n}{ }^{p}$ are the structure constants of the goup. Instead of these $k(k-1) / 2$ equations for $k$-dimensional group we have only one equation (2.9) to verify. 


\section{Transformation rules for Bondi integration functions}

Variations of the integration functions $\delta M, \delta N, \delta c$ can be found directly from variations of metric tensor components $\delta g_{00}, \delta g_{0 \Omega}, \delta g_{\Omega \Omega}$, respectively, where

$$
\begin{aligned}
\delta g_{i j}(x) & =g^{\prime}{ }_{i j}(x)-g_{i j}(x)= \\
& =g_{i k} \delta x^{k},{ }_{j}+g_{j k} \delta x^{k}, i \\
& -g_{i j, k} \delta x^{k} .
\end{aligned}
$$

To carry out the calculation, power series expansions of coordinate variations are needed. The result is uniquely determined by $(1.1),(1.2),(1.4)$ :

$$
\begin{aligned}
& \delta u=\tau, \\
& \delta \Omega=\omega+\tilde{\delta} \tau / r-c \partial \tau / r^{2}+c \bar{c} \tilde{\partial} \tau / 2 r^{3}, \\
& \delta r=-\left(\frac{\partial \tau}{\partial u}\right) r+\frac{1}{2} \tilde{\partial} \partial \tau+\left\{-\frac{1}{4} c ð \partial \tau-\frac{1}{2}(\partial c) \partial \tau+\text { c. c. }\right\} / r,
\end{aligned}
$$

where c.c. denotes the complex conjugate of the preceding expression in the brackets.

From (1.1), (3.1), (3.2) $\delta N, \delta M, \delta c$ can be calculated directly and we may try to give the result in the form $(2.3)$ :

$$
\begin{gathered}
\delta A=S_{A B}(\tau, \omega) B, \\
A ; B \in\{N, M, c, \bar{c}\} .
\end{gathered}
$$

(We hope that the use of the same symbols $A, B$ for function as well as for matrix indices will not cause any confusion.) When we carry out these calculations, we find that not the whole variations $\delta N$ and $\delta c$ can be given in the form of (3.3), (3.4). The outcome is

$$
\begin{aligned}
& \delta N=S_{N B} B-(\tilde{\delta} \tau) c \dot{\bar{c}} \text { where } \dot{\bar{c}} \equiv \frac{\partial \bar{c}}{\partial u}, \\
& \delta c=S_{c, c} c+\frac{1}{2} \tilde{\delta} \tilde{\delta} \tau .
\end{aligned}
$$

We see that the formula (3.3) may be retained if we add two more functions to (3.4), $c \dot{\bar{c}}$ and $I \equiv 1$. Then (3.3) will describe the transformation completely with

$$
A, B \in\{N, M, c \dot{\bar{c}}, c, \bar{c}, I\} .
$$

The nonvanishing components of the full $S_{A B}$ are the following:

$$
\begin{aligned}
& S_{N N}=S_{3,-1}(\tau, \omega), \\
& S_{N M}=-(\tilde{\partial} \tau), \\
& S_{N, c \dot{\bar{c}}}=-(\tilde{\partial} \tau), \\
& S_{N c}=\frac{1}{4}(\tilde{\partial} \tau) \partial \partial, \\
& S_{N \bar{c}}=-\frac{1}{4}(\tilde{\partial} \tau) \tilde{\partial} \tilde{\delta}, \\
& S_{M M}=S_{3,0}(\tau ; \omega) ; \\
& S_{M c}=-\frac{1}{2}(\delta \tau) \partial \frac{\partial}{\partial u}-\frac{1}{4}(\partial \partial \tau) \frac{\partial}{\partial u},
\end{aligned}
$$




$$
\begin{aligned}
& S_{M \bar{c}}=-\frac{1}{2}(\tilde{\partial} \tau) \tilde{\partial} \frac{\partial}{\partial u}-\frac{1}{4}(\tilde{\partial} \tilde{\partial} \tau) \frac{\partial}{\partial u} ; \\
& S_{c \dot{\bar{c}}, c \dot{\bar{c}}}=S_{3,0}(\tau, \omega) \text {, } \\
& S_{c \dot{\bar{c}}, \bar{c}}=\frac{1}{2}(\tilde{\delta} \tilde{\partial} \tau) \frac{\partial}{\partial u}, \\
& S_{c, c} i=S_{1,-2}(\tau, \omega) \text {, } \\
& S_{c I}=\frac{1}{2}(\tilde{\partial} \tilde{\tilde{}} \tau) \\
& S_{\bar{c}, \bar{c}}=S_{1,+2}(\tau, \omega) \text {, } \\
& S_{\bar{c} I}=\frac{1}{2}(ð ð \tau) \text {. }
\end{aligned}
$$

Here we have introduced the convention

$$
S_{v, s}(\tau, \omega)=-\tau \frac{\partial}{\partial u}+\frac{1}{2} \omega \partial_{s}+\frac{1}{2}-\tilde{\partial}_{s}-v\left(\frac{\partial \tau}{\partial u}\right)+\frac{s}{4}(\partial \omega-\tilde{\partial} \tilde{\omega}) .
$$

Our next task will be to employ the formalism of the previous section to prove that (3.6) forms a representation of the BMS group defined by (1.5).

\section{The proof that (3.6) forms a BMS representation}

We have to verify the basic commutation relation (2.9) in the case of (3.6). The procedure is straightforward and the outcome is positive, (3.6) in fact satisfies (2.9). Here we only refer to some peculiarities, and outline the main stages of the treatment.

First, we state that the use of complex function $\omega$ and edth operators $\delta, \bar{\delta}$ instead of $\vartheta, \varphi$ and derivatives with respect to them, considerably simplifies the presentation (3.6) of the matrix $S$, but at the same time requires some additional care. Direct calculation shows that despite the fact that $\omega$ does not transform according to any BMS representation, (2.6) still holds with

$$
S_{0}(\tau, \omega)=-\tau \frac{\partial}{\partial u}+\frac{1}{2} \omega \check{\partial}_{s}+\frac{1}{2}-\tilde{\partial}_{s}
$$

$s=-1,0,1$ when $S_{0}$ acts on $\omega, \tau$ or $\bar{\omega}$, respectively. Now for (3.8) it may be directly checked that

$$
\begin{gathered}
S_{v, s}\left(\tau_{1}, \omega_{1}\right) S_{v, s}\left(\tau_{2}, \omega_{2}\right)-1 \leftrightarrow 2= \\
=S_{v, s}\left(S_{0}\left(\tau_{1}, \omega_{1}\right) \tau_{2}, S_{0}\left(\tau_{1}, \omega_{1}\right) \omega_{2}\right)-1 \leftrightarrow 2,
\end{gathered}
$$

where the effect of the term $\frac{s}{4}\left(\check{\omega}_{\omega}-\tilde{\partial} \bar{\omega}\right)$ exactly compensates the term arising due to the noncommutativity of $\partial_{s}$ and $\tilde{\partial}_{s}$.

The expression (4.2) verifies the basic commutational relation (2.9) for diagonal elements of transformation matrix (3.6). Much more effort is needed to do the same for other elements. As an auxiliary quantity, it is useful to define (no summation over repeated indices)

$$
\begin{aligned}
K_{A B}\left(\tau_{1}, \omega_{1} ; \tau_{2}, \omega_{2}\right) & =S_{A A}\left(\tau_{1}, \omega_{1}\right) S_{A B}\left(\tau_{2}\right)+S_{A B}\left(\tau_{1}\right) S_{B B}\left(\tau_{2}, \omega_{2}\right)- \\
& -S_{A B}\left(S_{0}\left(\tau_{1}, \omega_{1}\right) \tau_{2}\right)-1 \leftrightarrow 2 .
\end{aligned}
$$


A rather lengthy expression may be deduced for $K$; containing commutators which are dealt in Appendix A, and then the desired result will be obtained:

$$
\begin{aligned}
& K_{N M}=K_{N, c \bar{c}}=K_{M c}=K_{c \dot{\bar{c}}, c}=K_{c I}=0, \\
& K_{N c}\left(\tau_{1}, \omega_{1} ; \tau_{2}, \omega_{2}\right)+ \\
& \quad+\left\{S_{N M}\left(\tau_{1}\right) S_{M c}\left(\tau_{2}\right)-1 \leftrightarrow 2\right\}=0, \\
& K_{N \bar{c}}\left(\tau_{1}, \omega_{1} ; \tau_{2}, \omega_{2}\right)+ \\
& \quad+\left\{S_{N M}\left(\tau_{1}\right) S_{M \bar{c}}\left(\tau_{2}\right)+S_{N, c \bar{c}}\left(\tau_{1}\right) S_{\left.c \dot{\bar{c}} \overline{\bar{c}}\left(\tau_{2}\right)-1 \leftrightarrow 2\right\}=0 .}\right.
\end{aligned}
$$

The outcome (4.2), (4.4) verifies the basic commutation relation (2.9) for the transformation (3.6), thus proving it to form a representation.

\section{APPENDIX A}

\section{Edth operators $\partial, \tilde{\delta}$}

$$
\text { Definitions: } \begin{aligned}
\tilde{\delta}_{s} & =-\frac{\partial}{\partial \vartheta}+s \cot \vartheta-(\mathrm{i} / \sin \vartheta) \frac{\partial}{\partial \varphi}, \\
\tilde{\delta}_{s} & =-\frac{\partial}{\partial \vartheta}-s \cot \vartheta+(\mathrm{i} / \sin \vartheta) \frac{\partial}{\partial \varphi} .
\end{aligned}
$$

Here $s$ is an integer determined by the spin weight of the function on which $\partial$ or $\tilde{\partial}$ acts. The complex conjugate of a function has the spin weight of the opposite sign. The spin weights for functions used in this paper are the following: -2 for $c,-1$ for $N, 0$ for $M$ and $\tau$. Function $\omega$ has no strictly defined spin weight, but in integrity with $\partial, \tilde{\partial}$ it may be treated as if its spin weight were -1 . The product of two functions has spin weight equal to the sum of their spin weights. Thus the Leibnitz rule for $\partial, \tilde{\partial}$ is derived from (A.1):

$$
\nearrow_{s+s^{\prime}}(A B)=\left(\nearrow_{s} A\right) B+A\left(\nearrow_{s^{\prime}} B\right)
$$

and analogically for $\tilde{\partial}$.

The operator $\partial$ raises and $\tilde{\delta}$ lowers the spin weight of the function by one. Thus their commutator must be defined as follows:

$$
[\tilde{\partial}, ð]_{s}=\tilde{\partial}_{s+1} \chi_{s}-\chi_{s-1} \tilde{\partial}_{s} .
$$

Taking into account (A.1),

$$
[\tilde{\partial}, \check{\partial}]_{s}=2 s .
$$

With the help of (A.2), commutators of any $\partial, \tilde{\tilde{\delta}}$ combinations may be calculated, with some extra care for spin weight. For example

$$
[\tilde{\delta}, \partial \tilde{\partial}]_{s}=\partial_{s}[\tilde{\delta}, \tilde{\partial}]_{s}+[\tilde{\partial},]_{s+1} \partial_{s}=2(1+2 s) \check{\partial}_{s} .
$$




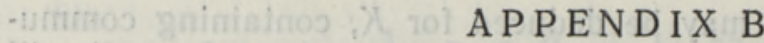

\section{$\tau$ and $\omega$ for Poincaré transformations}

1. The Lorentz-transformations.

$\left(\tau_{i j}, \omega_{i j}\right.$ denoting rotation in $i j$-plain of the Minkowski space)

$$
\begin{array}{ll}
\tau_{01}=u \sin \vartheta \cos \varphi, & \omega_{01}=-\cos \vartheta \cos \varphi-\mathrm{i} \sin \varphi, \\
\tau_{02}=u \sin \vartheta \sin \varphi, & \omega_{02}=-\cos \vartheta \sin \varphi+\mathrm{i} \cos \varphi, \\
\tau_{03}=u \cos \vartheta, & \omega_{03}=\sin \vartheta \\
\tau_{\alpha \beta}=0 ; & \omega_{\alpha \beta}=-\varepsilon_{\alpha \beta \sigma} i \omega_{0 \sigma} .
\end{array}
$$

2. Translations.

$\left(\tau_{k}, \omega_{k}\right.$ denoting translation in the direction of $k$-axis)

$$
\begin{aligned}
& \tau_{0}=1, \\
& \tau_{1}=\sin \vartheta \cos \varphi, \\
& \tau_{2}=\sin \vartheta \sin \varphi \\
& \tau_{3}=\cos \vartheta .
\end{aligned}
$$

The rest of $u$-independent $\tau(\vartheta, \varphi)$ describes supertranslations.

\section{REFERENCES}

1. Winicour, J. In: General Relativity and Gravitation (ed. by A. Held), vol. 2. New York, Plenum Publishing, 1980.

2. Ko, M., Ludwigsen, M., Newman, E. T. Phys. Rep., 71, № 2, 51-139 (1981).

3. Sachs, $R$. Proc. Roy. Soc., A270, № 1340, 103-126 (1962).

4. DeWitt, B. S. In: Relativity, Groups and Topology. New York, Gordon \& Beach Sci. Publ., 1964.

Academy of Sciences of the Estonian SSR, Institute of Astrophysics and Atmospheric Physics

Received Apr. 4, 1986

\section{A. UNT}

\section{BONDI INTEGREERIMISFUNKTSIOONIDE TEISENEMISEESKIRJAD}

On leitud Bondi integreerimisfunktsioonide teisenemiseeskirjad (3.6) infinitesimaalsetel BMS teisendustel ja tuletatud tingimus (2.9), mille abil tôestatakse, et kujul (3.6) kirjapandud teisendus moodustab BMS rühma esituse.

\section{A. УНT}

\section{ТРАНСФОРМАЦИОННЫЕ ПРАВИЛА ДЛЯ ФУНКЦИИ ИНТЕГРИРОВАНИЯ} Бонди

Найдены трансформационные правила (3.6) для функций интегрирования Бонди при инфинитезимальных преобразованиях БМС. Выведено условие (2.9), с помощью которого доказано, что преобразование, написаншое в форме (3.6), является представлением группы БМС. 